\title{
Anabases
}

ANABASES Traditions et réceptions de l'Antiquité

17 | 2013

Varia

\section{Simon HORNBLOWER, Antony SPAWFORTH et al., The Oxford Classical Dictionary}

\section{Tomás Fernández Caracciolo Conicet}

\section{(2) OpenEdition}

\section{Journals}

Édition électronique

URL : http://journals.openedition.org/anabases/4262

DOI : 10.4000/anabases.4262

ISSN : 2256-9421

\section{Éditeur}

E.R.A.S.M.E.

\section{Édition imprimée}

Date de publication : 1 mars 2013

Pagination : 281-282

ISSN : 1774-4296

\section{Référence électronique}

Tomás Fernández Caracciolo Conicet, « Simon hornblower, Antony spawforth et al., The Oxford Classical Dictionary ", Anabases [En ligne], 17 | 2013, mis en ligne le 01 avril 2013, consulté le 22 septembre 2020. URL : http://journals.openedition.org/anabases/4262 ; DOI : https://doi.org/10.4000/anabases 4262

Ce document a été généré automatiquement le 22 septembre 2020

(c) Anabases 


\title{
Simon HORNBLOWER, Antony SPAWFORTH et al., The Oxford Classical Dictionary
}

\author{
Tomás Fernández Caracciolo Conicet
}

\section{RÉFÉRENCE}

Simon HORNBLOWER, Antony SPAWFORTH et al., The Oxford Classical Dictionary, Oxford-New York, Oxford University Press, 2012 (4 éd.), 1648 p.

100 livres / ISBN 978-0-19-954556-8.

1 Les éditeurs de l'OCD déclarent - dès la troisième édition - que « as an authoritative onevolume guide to all aspects of the ancient world, $O C D$ has no competitor in any language " (Preface to the third edition, p. ix), ainsi que " no other single-volume work of reference remotely approaches $O C D$ in the sheer quality of factual detail contained» (ibid.). Qu'est-ce qu'une recension pourrait ajouter, si les éditeurs eux-mêmes sont aussi emphatiques dans les louanges de leur ouvrage ? La troisième édition incorporait mille nouvelles entrées ; cette quatrième édition en comporte moins de cent, entre « new and replacement entries ». Par conséquent, elle est plutôt une révision de la précédente (elle-même révisée) qu'une véritable nouvelle édition.

2 Il pourrait s'avérer instructif de signaler quelques particularités relevées au hasard des premières lectures. Par exemple, il y a une entrée sur Anthologies, Latin, tandis qu'il en manque une pour les anthologies grecques, pourtant non moins importantes; le lecteur trouvera seulement une entrée portant sur l'Anthologia Graeca, sans aucune référence à l'Anthologion de Jean Stobée. Il y a aussi des jugements subjectifs dont la pertinence dans un dictionnaire encyclopédique est contestable; ainsi, on lit que Cicéron "grotesquely calls "bold metaphor" " une image fréquente en son temps, sitire agros (dans Metaphor and simile); est-il opportun d'estimer grotesque un usage semblable? Il est aussi déclaré que l'Anthologia Graeca « is one of the great books of European 
literature, a garden containing the flowers and weeds of fifteen hundred years of Greek poetry, from the most humdrum doggerel to the purest poetry » (dans Anthology, Greek). $\mathrm{Au}$-delà du pardonnable verbiage, la tendance à taxer un ouvrage de «bon» ou « mauvais », « humdrum doggerel » ou " purest poetry », est quelque peu gênante et découle d'une conception de la littérature que nombre de spécialistes estiment aujourd'hui périmée.

De même, des approches méthodologiques qui semblent d'un autre temps subsistent dans cette nouvelle édition. Dans l'entrée Calpurnius Siculus, on lit par exemple que le berger Corydon « is generally identified with Calpurnius »; pourtant, à l'heure actuelle, on hésiterait à identifier ainsi un personnage et un auteur. Du reste, on peut détecter une certaine inconsistance dans des catégories pourtant fondamentales. Il est ainsi sous-entendu que les classes supérieures existaient pleinement dans l'Antiquité (on parle d' " upper-class disdain for personal involvement in trade and exchange », dans Economic theory [Greek]), tandis qu'ailleurs cette assomption est très raisonnablement mise en question (dans Class struggle).

4 L'OCD ne manque pas non plus d'affirmations qui, en prétendant mettre fin à un malentendu populaire, lui en substituent un autre qui pourrait être considéré comme plus grave: "The preoccupation with plagiarism over many centuries serves as a reminder that, contrary to some modern misstatements, ancient literature, especially poetry, was expected to be "new". Certainly, many writers, Greek and Roman, are anxious to assert the necessity of originality or their own claim to it »(dans Plagiarism.) Ne risque-t-on pas, avec des phrases semblables, de faire croire que les anciens avaient une conception de l'«originalité » compatible avec celle des romantiques? Les

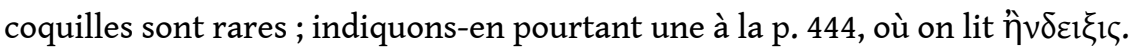

En un mot, l'OCD ne prend pas toujours en considération les acquis de la philologie classique contemporaine, de la linguistique ou de l'historiographie. En outre, il serait sans doute plus pratique d'avoir un $O C D$ en ligne, avec une actualisation plus régulière. Ceci permettrait aussi d'inclure des illustrations et des cartes géographiques que les éditeurs, pour des raisons de moins en moins convaincantes, refusent d'ajouter à cette nouvelle édition.

Même si cette recension a exprimé certaines réserves sur la nouvelle édition de l'ocD, il convient de signaler qu'elle reste précieuse pour ceux qui étudient l'Antiquité classique. Cela va sans dire : il y a des articles purement et simplement excellents, tout aussi utiles pour le spécialiste que pour l'amateur. On peut pourtant espérer que les éditeurs futurs s'apercevront de la nécessité d'opérer des changements beaucoup plus substantiels si l'oCD doit garder une place centrale dans le monde de l'érudition à l'époque de la New Pauly Online... et de Wikipédia. 


\section{AUTEURS}

TOMÁS FERNÁNDEZ CARACCIOLO CONICET

Universidad de Buenos Aires

Katholieke Universiteit Leuven

tomas.fernandez@arts.kuleuven.be 